\title{
Mecanismo Extensor da Mão: Desvendando a Anatomia e Avaliação por Métodos de Imagem
}

\section{Hand extensor mechanism: Unraveling the anatomy and imasing analysis}

\author{
Marina Celli Francisco ${ }^{(1)}$, Je Hoon Yang $^{(2)}$, Felipe Trentin Neves ${ }^{(3)}$, Fabiano Celli Francisco $^{(4)}$, \\ Jamil Natour ${ }^{(5)}$, Artur da Rocha Correa Fernandes ${ }^{(6)}$
}

Médicos experientes apresentam grande dificuldade no exame físico de pacientes com suspeita de lesão dos tendões do antebraço e da mão. Estudos relatam até $20 \%$ de atrasos no diagnóstico, resultando em morbidade que poderia ser prevenida e evitada. Em atendimento de emergência, o diagnóstico clínico correto é realizado em apenas $64 \%$ dos $\operatorname{casos}^{(1)}$.

Pode ser difícil diferenciar ruptura tendínea de aderência do tendão ou desordens neurológicas. O local e a dimensão da ruptura podem não ser evidentes ao exame físico, especialmente em casos de lesão degenerativa de tendão com distúrbio motor incompleto, em que o edema local e a sensibilidade aumentada podem não estar presentes $^{(2)}$.

Desse modo, os métodos de imagem desempenham importante papel no auxílio diagnóstico das lesões dos músculos extensores da mão, ajudando a direcionar a terapêutica mais adequada em função da causa.

\section{ASPECTOS ANATÔMICOS}

Os movimentos extensores da mão são realizados por dois conjuntos de músculos, a saber: os extensores intrínsecos, ou seja, aqueles que possuem sua origem e inserção na própria mão, e os extrínsecos, cuja origem é no cotovelo e antebraço.

Os músculos extensores extrínsecos são responsáveis pela extensão das articulações metacarpofalangianas e interfalangianas, compreendendo o extensor dos dedos, o extensor do indicador e o extensor do dedo mínimo ${ }^{(3)}$.

Já os músculos extensores intrínsecos são os lumbricóides e interósseos, que, além de estenderem as articu- lações interfalangianas, também contribuem na flexão das metacarpofalangianas ${ }^{(3)}$.

Para melhor compreensão da musculatura da mão e da topografia das lesões, Kleinert e Verdan ${ }^{(4)}$ propuseram um sistema de classificação em zonas (Figura 1):

- zona I: articulação interfalangiana distal;

- zona II: falange média;

- zona III: articulação interfalangiana proximal;

- zona IV: falange proximal;

- zona V: articulação metacarpofalangiana;

- zona VI: dorso da mão;

- zona VII: compartimentos extensores do punho (Figura 2);

- zona VIII: músculos extensores extrínsecos (Figuras 3 e 4$)$.

As zonas possuem grande importância para se localizar corretamente a região anatômica da lesão, o que, associado ao tipo da lesão e à presença de deformidades, direciona o diagnóstico e o procedimento cirúrgico a serem realizados.

\section{AVALIAÇÃo POR MÉTODOS DE IMAGEM}

\section{RADIOGRAFIA CONVENCIONAL}

Durante muitas décadas, a radiografia de baixa quilovoltagem e a xerorradiografia eram as únicas técnicas de imagem aplicáveis aos tendões. Apesar de poderem delinear os tendões, particularmente quando envoltos por gordura, essas técnicas falhavam em demonstrar a sua estrutura interna. Entretanto, ainda são boas modalidades para a documentação de pequenas calcificações em tendões ou lesões ósseas ${ }^{(5)}$.

3. Médico-residente de Cirurgia Geral do Hospital Ipiranga (SP).

4. Mestre em Radiologia Clínica pela Universidade Federal do Rio de Janeiro e médico radiologista do Hospital de Caridade São Braz de Porto União (SC)

5. Professor adjunto da Disciplina de Reumatologia da Unifesp.

6. Professor adjunto do Departamento de Diagnóstico por Imagem da Unifesp.

Endereço para correspondência: Artur da Rocha Correa Fernandes, DDI/Unifesp, Rua Botucatu, 740, 04023-900, São Paulo, SP, Brasil, e-mail:artur_personal@yahoo.com.br 


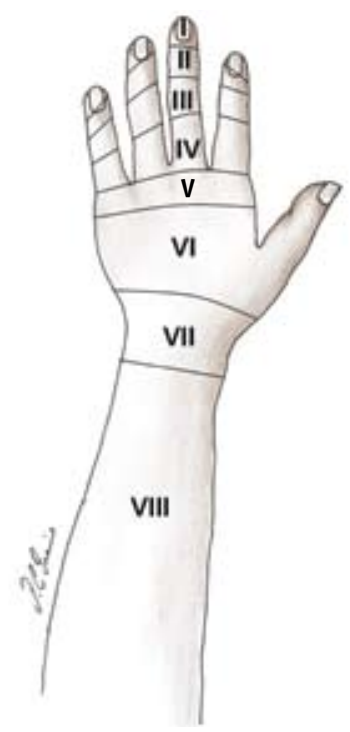

Figura 1 - Zonas do antebraço. Esquema da vista dorsal do antebraço mostrando a classificação de Verdan das zonas que dividem os músculos extensores da mão e antebraço: I. Articulação interfalangiana distal; II. Falange média; III. Articulação interfalangiana proximal; IV. Falange proximal; V. Articulação metacarpofalangiana; VI. Dorso da mão; VII. Compartimento extensor do punho; VIII. Músculos extensores extrínsecos.

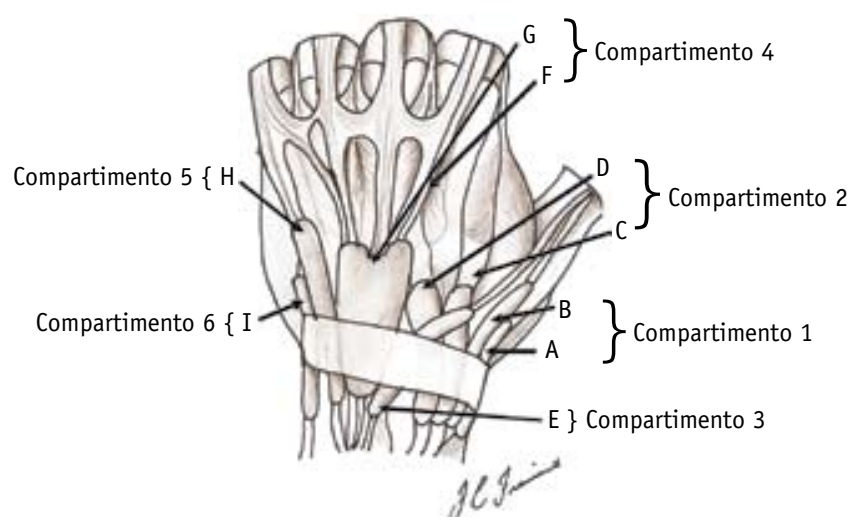

Figura 2 - Compartimentos da zona VII: A. Abdutor longo do polegar; B. Extensor curto do polegar; C. Extensor longo do carpo; D. Extensor curto do carpo; E. Extensor longo do polegar; F. Extensor do indicador; G. Extensor dos dedos; H. Extensor do dedo mínimo; I. Extensor ulnar do carpo.

\section{ULTRA-SONOGRAFIA}

Recentes avanços na tecnologia dos transdutores propiciaram a visualização de imagens com maior nitidez das estruturas superficiais. Qualidades como boa resolução espacial, velocidade do exame, baixo custo, não-utilização de radiação ionizante, além de ser um método dinâmico e não invasivo, podendo ser utilizado em avaliação seriada, conferem especial importância à ultra-sonografia na avaliação dos músculos extensores da mão e do antebraço (Figuras 5 e 6 ).

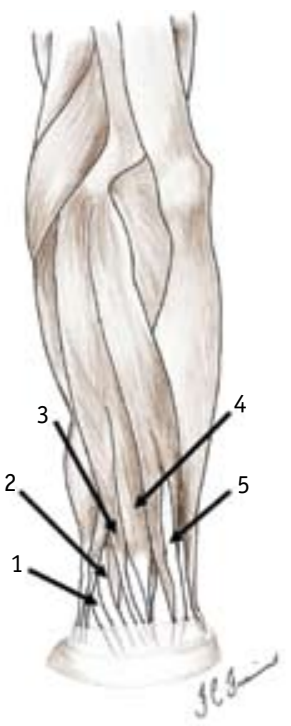

Figura 3 - Músculos extensores superficiais do antebraço. Vista póstero-lateral. 1. Extensor radial longo do carpo; 2. Extensor radial curto do carpo; 3. Extensor dos dedos; 4. Extensor do dedo mínimo; 5. Extensor ulnar do carpo.

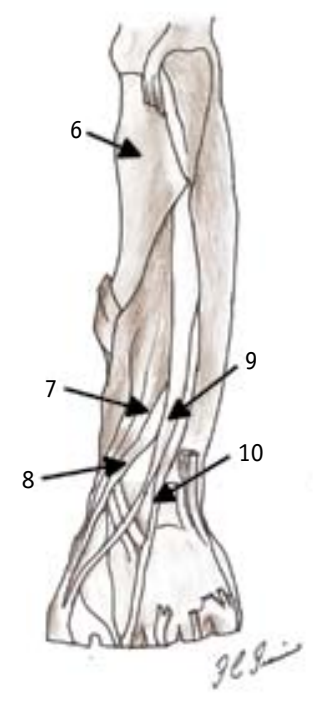

Figura 4 - Músculos extensores profundos do antebraço. Vista póstero-lateral após a remoção dos músculos extensores superficiais. 6. Supinador; 7. Abdutor longo do polegar; 8. Extensor curto do polegar; 9. Extensor longo do polegar; 10. Extensor do indicador.

No entanto, exige um profissional treinado e familiarizado para sua realização ${ }^{(6)}$.

Processos patológicos como proliferação sinovial e derrame são características das sinovites, com fácil diagnóstico à ultra-sonografia ${ }^{(7)}$, assim como tendinite, tendinopatia $\mathrm{e}$ rupturas completas ${ }^{(8)}$. 
Rupturas parciais apresentam sensibilidade e especificidade de $33 \%$ e $89 \%$, respectivamente, com acurácia de $75 \%^{(2)}$.

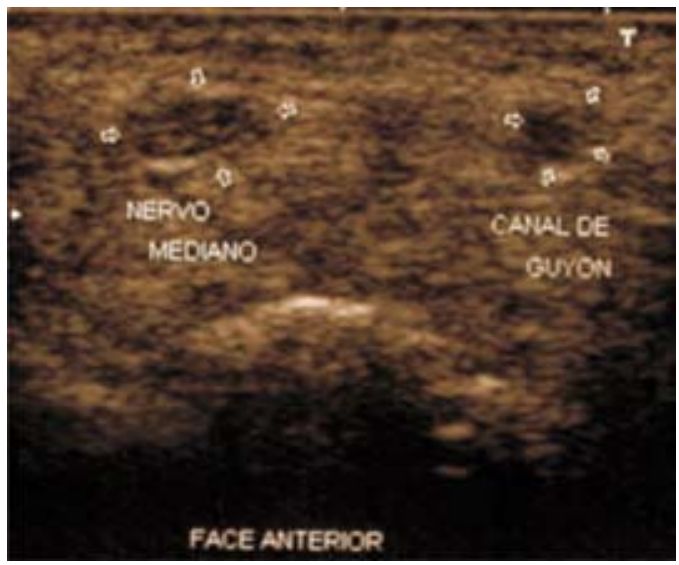

Figura 5 - Ultra-sonografia do punho. Vista axial. Nervo mediano (setas) e canal de Guyon (setas).

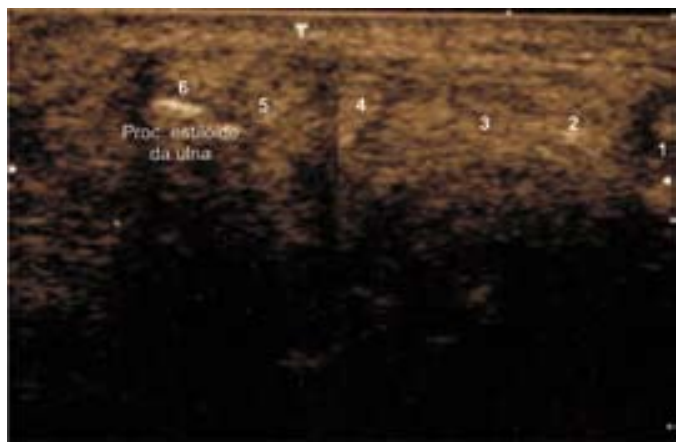

Figura 6 - Ultra-sonografia do punho. Vista axial. Compartimentos do punho. 1. Compartimento 1 do punho (abdutor longo do polegar e extensor curto do polegar); 2. Compartimento 2 do punho (extensor longo do carpo e extensor curto do carpo); 3 . Compartimento 3 do punho (extensor longo do polegar); 4. Compartimento 4 do punho (extensor do indicador e extensor dos dedos); 5. Compartimento 5 do punho (extensor do dedo mínimo); 6 . Compartimento 6 do punho (extensor ulnar do carpo).

\section{TOMOGRAFIA COMPUTADORIZADA}

Os ligamentos do punho e mão podem ser visualizados por tomografia computadorizada multidetectores e ressonância magnética. Na avaliação dos ligamentos do dorso da mão, a tomografia, associada à artrografia, mostrou-se mais acurada na deteç̧ão de lesões ( $83 \%$ versus $78 \%$ ) e apresentou menor variação interobservadores. Todavia, na análise dos ligamentos palmares e centrais, a literatura mostra performance semelhante entre os $\operatorname{métodos}^{(9)}$.
A tomografia computadorizada apresenta importante papel na detecção de fraturas ósseas que podem estar associadas a lesões musculares, detectando lesões ocultas à radiografia ou falsos-positivos. Além disso, nos atuais aparelhos com multidetectores, o membro pode ser mantido em posição de menor desconforto para o paciente e, posteriormente, por meio de reconstruções multiplanares, obtidas por pós-processamento das imagens fontes, obtémse análise mais detalhada das áreas de maior interesse ${ }^{(10)}$.

Clínicos e cirurgiões apresentam maior facilidade de compreender as lesões do antebraço e mão mediante reformatações de tomografia computadorizada e, com isso, realizam o tratamento clínico ou a programação cirúrgica ${ }^{(11)}$ (Figuras 7 a 9).

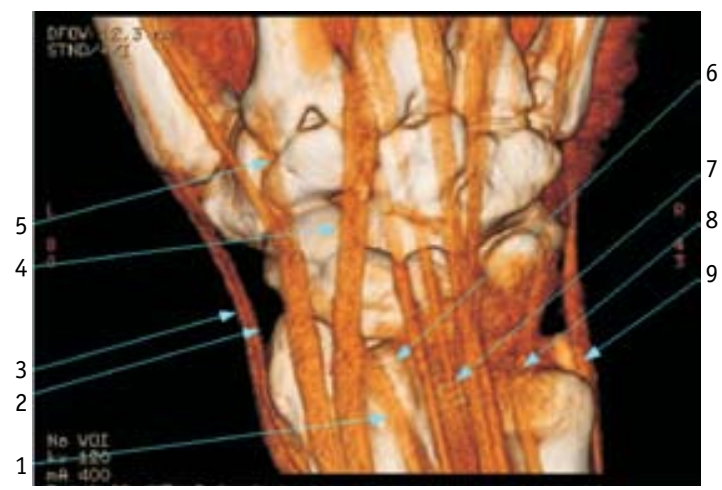

Figura 7 - Reformatação 3D de tomografia computadorizada. Vista dorsal do punho. 1. Extensor longo do polegar; 2. Extensor curto do polegar; 3. Abdutor longo do polegar; 4. Extensor radial longo do carpo; 5. Extensor radial curto do carpo; 6. Extensor do indicador; 7. Extensor dos dedos; 8. Extensor do dedo mínimo; 9. Extensor ulnar do carpo.

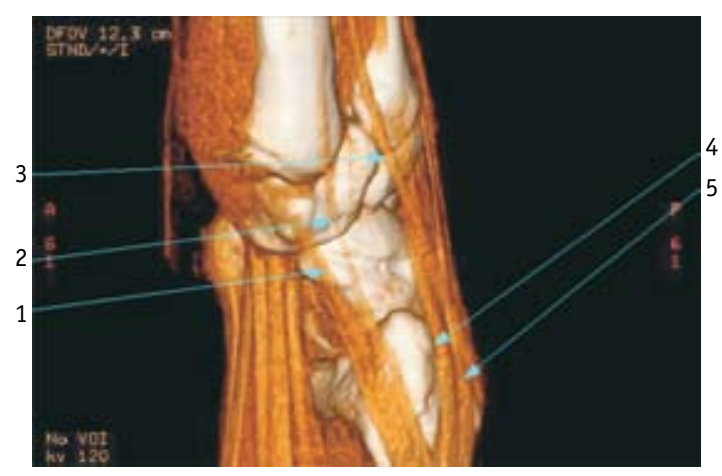

Figura 8 - Reformatação 3D de tomografia computadorizada. Vista da região medial do punho. 1. Abdutor longo do polegar; 2. Extensor curto do polegar; 3. Extensor longo do polegar; 4. Extensor radial longo do carpo; 5. Extensor radial curto do carpo. 


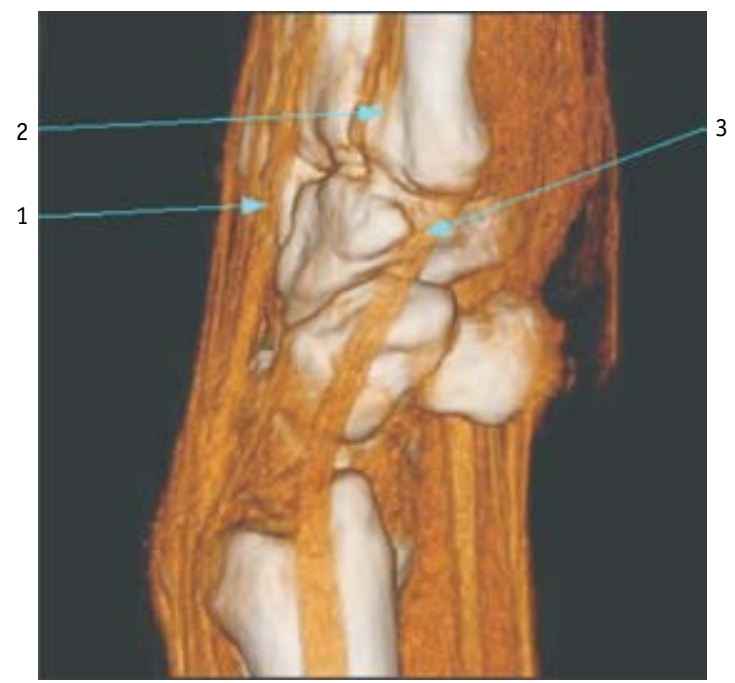

Figura 9 - Reformatação 3D de tomografia computadorizada. Vista da região lateral do punho. 1. Extensor dos dedos; 2. Extensor do dedo mínimo; 3. Extensor ulnar do carpo.

\section{RESSONÂNCIA MAGNÉTICA}

A ressonância magnética $(\mathrm{RM})$ não utiliza radiação ionizante. No entanto, apresenta alto custo e necessita de radiologistas habituados à avaliação musculoesquelética para sua interpretação. Apresenta boa resolução espacial e demonstra com precisão a relação anatômica de ligamentos, tendões, músculos, ossos e articulações (Figuras 10 a 12).

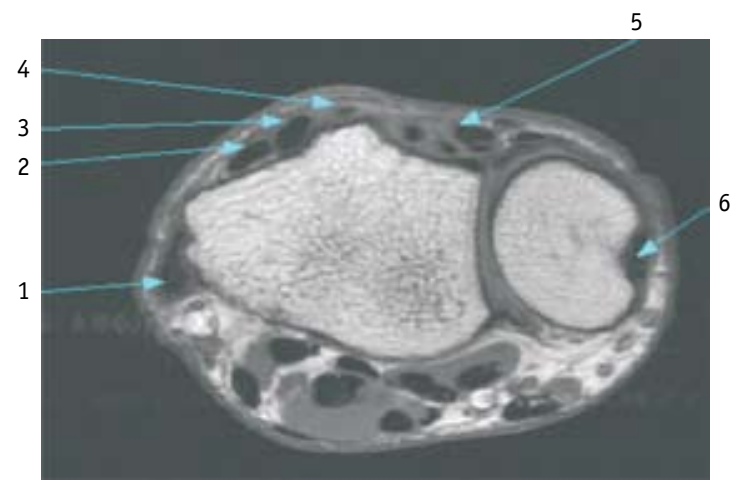

Figura 10 - Cortes axiais de ressonância magnética do punho. 1. Abdutor longo do polegar; 2. Extensor radial longo do carpo; 3. Extensor radial curto do carpo; 4. Extensor do indicador; 5. Extensor dos dedos; 6. Extensor ulnar do carpo.

O método mostra com clareza rupturas tendíneas, tanto completas quanto parciais, e precisa sua localização. É particularmente útil na avaliação do sistema extensor quando o exame clínico é duvidoso, principalmente na fase aguda

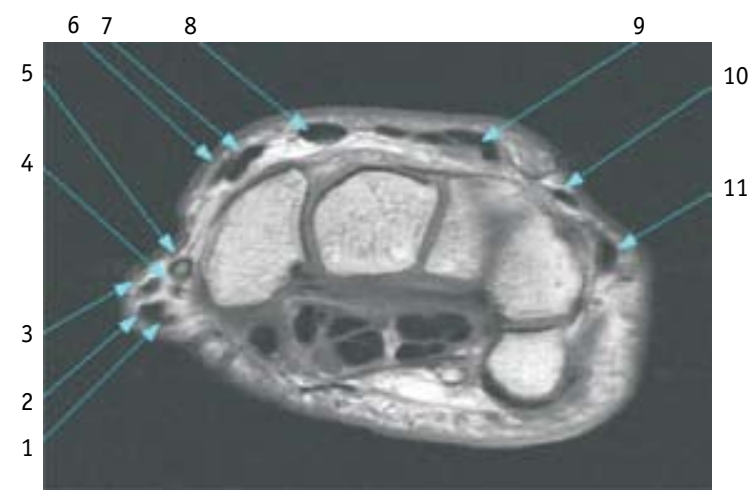

Figura 11 - Cortes axiais de ressonância magnética do punho. 1. Abdutor longo do polegar; 2. Extensor curto do polegar; 3. Extensor longo do polegar; 4. Nervo radial; 5 . Artéria radial; 6 . Extensor do indicador; 7. Extensor radial longo do carpo; 8. Extensor radial curto do carpo; 9. Extensor dos dedos; 10. Extensor do dedo mínimo; 11. Extensor ulnar do carpo.

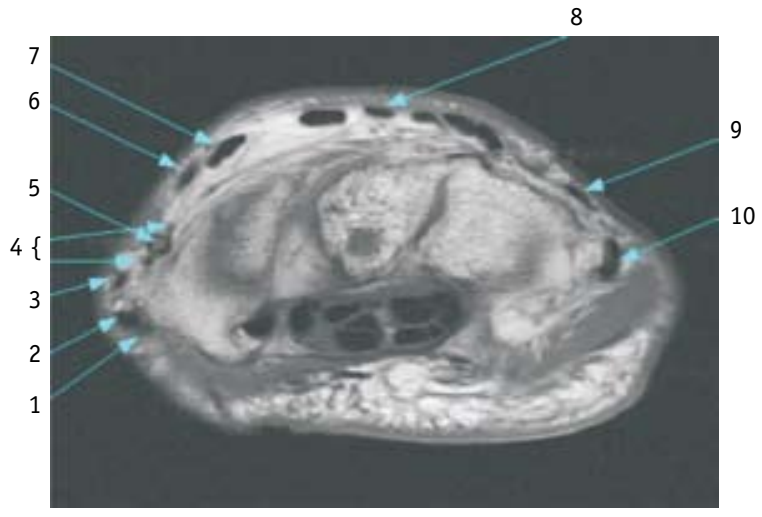

Figura 12 - Cortes axiais de ressonância magnética do punho. 1. Abdutor longo do polegar; 2. Extensor curto do polegar; 3. Extensor longo do polegar; 4. Nervo radial; 5. Artéria radial; 6. Extensor radial longo do carpo; 7. Extensor radial curto do carpo; 8. Extensor dos dedos; 9. Extensor do dedo mínimo; 10. Extensor ulnar do carpo.

da lesão. Fornece também importantes informações sobre o número de tendões envolvidos em lesões complexas e identifica variações anatômicas ${ }^{(12)}$.

Nas lesões em articulações, a RM é crucial na avaliação da extensão da lesão para ligamentos de suporte e cápsulas articulares, o que pode representar indicação cirúrgica ${ }^{(12)}$.

Rupturas parciais apresentam sensibilidade e especificidade de $27 \%$ e $83 \%$, respectivamente, com acurácia de $69 \%(2)$.

\section{CONCLUSÃO}

Deve-se iniciar a investigação diagnóstica com a realização de radiografia simples, no intuito de avaliar os 
ossos e partes moles, associada à ultra-sonografia, por apresentar boa precisão diagnóstica, pelo seu baixo custo, fácil acesso e por não utilizar radiação ionizante. Se esses exames obtiverem resultados normais e a suspeita clínica for baixa, pode-se interromper a investigação diagnóstica.

\section{REFERÊNCIAS}

1. Patel J, Coull R, Harris PA, Percival NJ: Hand lacerations: an audit of clinical examination. J Hand Surg (Br) 23B: 482-4, 1998.

2. Swen WAA, Jacobs JWG, Hubach PCG, Klasens JH, Algra PR, Bijlsma JWJ: Comparison of sonography and magnetic resonance imaging for the diagnosis of partial tears of finger extensor tendons in rheumatoid arthritis. Rheum 39: 55-62, 2000.

3. Ragheb D, Stanley A, Gentili A, Hughes T, Chung CB: MR imaging of the finger tendons: normal anatomy and commonly encountered pathology. Eur J Radiol 56(3): 296-306, 2005.

4. Kleinert HE, Verdan C: Report of the Committee on Tendon Injuries. J Hand Surg [Am] 8: 794-8, 1983.

5. Quintero SH, Quintero SO, Becerra E, Rigo SC: Xerorradiografia en ortopedia y traumatologia. Rev Soc Méd.-Quir Hosp Emerg Pérez de León 19(1): 75-82, 1984.

6. Lee JC, Healy JC: Normal sonographic anatomy of the wrist and hand. RadioGraphics 25: 1577-90, 2005.
Caso os exames estejam normais e seja alta a suspeita clínica ou se estiverem alterados, com informações discrepantes em relação à semiologia, deve-se prosseguir a investigação com tomografia computadorizada ou ressonância magnética, preferencialmente com esta última, quando a suspeita clínica estiver relacionada a lesões tendíneas e ligamentares.

7. Grassi W, Tittarelli E, Blasetti P, Pirani O, Cervini C: Finger tendon involvement in rheumatoid arthritis. Evaluation with highfrequency sonography. Arthritis Rheum 38: 786-94, 1995.

8. Chemm RK, Kaplan PA, Dussault RG: Ultrasonography of the musculoskeletal system. Radiol Clin North Am 32: 275-89, 1994.

9. Schmid MR, Schertler T, Pfirrmann CW, et al.: Interosseous ligament tears of the wrist: comparison of multi-detector row CT arthrography and MR imaging. Radiology 237: 1008-13, 2005.

10. Groves AM, Cheow HK, Balan KK, Bearcroft PW, Dixon AK: 16 detector multislice CT versus skeletal scintigraphy in the diagnosis of wrist fractures: value of quantification of $99 \mathrm{Tcm}-$ MDP uptake. Br J Radiol 78(933): 791-5, 2005.

11. Sunagawa T, Ishida O, Ishiburo M, Suzuki O, Yasunaga Y, Ochi $\mathrm{M}$ : Three-dimensional computed tomography imaging - Its applicability in the evaluation of extensor tendons in the hand and wrist. J Comput Assist Tomogr 29: 94-8, 2005.

12. Clavero JA, Golano P, Farinas O, Alomar X, Monill JM, Esplugas $\mathrm{M}$ : Extensor mechanism of the fingers: $\mathrm{MR}$ imaging-anatomic correlation. RadioGraphics 23: 593-611, 2003. 